\title{
LA EVALUACIÓN POR COMPETENCIAS EN EDUCACIÓN FÍSICA Y EL PROCESO DE CONSTRUCCIÓN DE UNA RÚBRICA
}

\author{
Jorge Agustín Zapatero Ayuso \\ Universidad Politécnica de Madrid \\ María Dolores González Rivera \\ Universidad de Alcalá \\ Antonio Campos Izquierdo \\ Universidad Politécnica de Madrid
}

RESUMEN: Desde su incorporación a la normativa del sistema educativo español, las competencias se han convertido en un referente curricular, planteándose como los elementos que vertebran la evaluación y promoción (Ley Orgánica 8/2013, de 9 de diciembre, para la mejora de la calidad educativa). El hecho de incluir las competencias en el currículo exigió cambios en el desempeño docente, cuya labor debe procurar el desarrollo de una evaluación formativa y basada en competencias. No obstante, los estudios evidencian que estas evaluaciones no llegan a desarrollarse en el día a día de los centros educativos. Se advierte de la falta de preparación del profesorado, el escaso asesoramiento de las administraciones y la falta de acuerdos en los centros educativos. En este contexto, este estudio pretende ser una guía para que el profesorado en general, y en particular el de Educación Física, pueda plantear y llevar a cabo evaluaciones por competencias mediante el diseño de rúbricas que valoren el grado de adquisición de las competencias en sus alumnos.

PALABRAS CLAVE: Competencias clave, evaluación formativa, Educación Física, formación del profesorado.

\section{THE ASSESSMENT BASED ON COMPETENCIES IN PHYSICAL EDUCATION AND THE PROCESS OF BUILDING A RUBRIC}

\footnotetext{
ABSTRACT: Since its incorporation into the law of the Spanish educational system, competencies have become a curricular reference, considering the elements that underpin the assessment and promotion (Organic Law 8/2013, of December 9, to improve educational quality). The fact of including competencies in the curriculum requires changes in teacher performance,
} 
whose labour should tend to develop models of formative assessment based on competencies. However, studies show that these assessments do not quite develop in the daily life of schools. It warns of the lack of preparation of teachers, poor advice administrations and lack of agreements in schools. In this context, this study is intended as a guide for teachers in general and, in particular the Physical Education teachers can raise and carry out assessments based on competencies trough designing rubrics to rate the degree of development of competencies in their students.

KEYWORDS: Key competencies, formative assessment, Physical Education, teacher training.

Recibido: 01/10/2016

Aceptado: 14/07/2016

Correspondencia: Jorge Agustín Zapatero Ayuso, Facultad de Educación-Centro de Formación del Profesorado, Universidad Complutense de Madrid. C/ Rector RoyoVillanova, s/n, Madrid (Madrid).Email: jzapater@ucm.es.

\section{EL CONCEPTO "COMPETENCIA" Y SU INCLUSIÓN EN EL PROCESO DE EVALUACIÓN}

El término competencia ha sido utilizado por disciplinas como la psicología, la dirección de recursos humanos, la sociología o las propias ciencias de la educación (Tobón, Rial, Carretero y García, 2006). Este hecho ha sustentado una complejidad terminológica y una baja uniformidad sobre lo que se entiende por competencia y los componentes que la integran (Ellström y Kock, 2008; Illeris, 2008; Zabala y Arnau, 2007).

Desde una perspectiva educativa, el proyecto DeSeCo de la OCDE definió la competencia como la capacidad para responder exitosamente a exigencias complejas en un contexto particular, movilizando conocimientos y aptitudes prácticas y componentes sociales y comportamentales como actitudes, emociones, valores y motivaciones (Rychen y Salganik, 2004). El Consejo Europeo (2006) entiende por competencia "una combinación de conocimientos, capacidades y actitudes adecuados para una determinada situación" (p. 13). Para Perrenoud (2004), el concepto de competencia "representa una capacidad de movilizar varios recursos cognitivos para hacer frente a un tipo de situaciones" (p. 11). Siguiendo a Moya y Luengo (2011), las competencias constituyen la forma en que las personas logran movilizar todos sus recursos (cognitivos, afectivos, sociales...) para lograr el éxito en la resolución de una tarea en un contexto definido.

De acuerdo con estos planteamientos, el concepto de competencia engloba varios componentes que deben movilizarse para la resolución de problemas. De hecho, frecuentemente la competencia se ha definido en términos de habilidades cognitivas (o conocimientos), habilidades perceptivo-motrices (o destrezas), factores afectivos (o actitudes y valores) y rasgos de personalidad (Baartman y Bruijn, 2011; Ellström y Kock, 2008; Rieckmann, 2012). En consecuencia, el desempeño competente supone la movilización de saberes ("saber", "saber hacer" y "saber ser") para afrontar situaciones de diferente índole con éxito (Orden ECD/65/2015, p. 6987). 
En lo que respecta a las competencias clave, éstas se caracterizan por estar al alcance de todo el mundo, ser transferibles a una amplia gama de situaciones de vida, contribuir al "aprendizaje a lo largo de la vida" y ser la base para el desarrollo de otras competencia más específicas en el futuro (Tiana, Moya y Luengo, 2011). Además, estas competencias son aquellas en las que se sustenta el desarrollo personal, la ciudadanía activa, la cohesión social y la empleabilidad (Consejo Europeo, 2006; Orden ECD/65/2015).

No obstante, adoptar el concepto de competencias en el discurso educativo va más allá de un debate conceptual. Su incorporación supone un replanteamiento del contenido, que no posee un valor en sí mismo, sino un valor funcional, cuya importancia reside en la aplicación del contenido en una situación concreta (Tejada, 2012). Asimismo, la rápida evolución y el frecuente cambio del contenido en la actual sociedad del conocimiento tienen consecuencias sobre la finalidad de la enseñanza, siendo ésta "el aprendizaje a lo largo de la vida". El objetivo es formar alumnos autónomos, flexibles y responsables, para lo cual es necesario otorgarles protagonismo en el proceso de enseñanza y aprendizaje (Illeris, 2008; Tejada, 2012).

Concretamente en este trabajo se sigue la concepción curricular de las competencias clave (Orden ECD/65/2015). En este sentido, la competencia es el conjunto de conocimientos, habilidades y actitudes (observables), que se ponen en acción sobre una base de valores, rasgos de personalidad y capacidades (no observables), para resolver una situación-problema con éxito en el contexto de un centro educativo y/o en el entorno cercano al alumno. Además, las competencias clave son aquellas que adquieren un carácter transversal a todas las asignaturas y que dotan de coherencia y globalidad a la intervención docente en un centro. En este sentido, todas ellas pueden y deben ser desarrolladas y evaluadas desde todas las asignaturas y, al mismo tiempo, son nexos para articular el trabajo entre materias. Este planteamiento conlleva atender a unas consideraciones para proponer una estrategia evaluativa coherente con el concepto de competencia:

- Las competencias clave son elementos curriculares y, por tanto, para programar su contribución y evaluación, se debe atender a las orientaciones normativas que las fundamentan (Consejo Europeo, 2006; Orden ECD/65/2015).

- Lascompetencias son transversalesy sepueden abordar desdetodas las asignaturas, advirtiéndose una doble consecuencia. Por un lado, la contribución y evaluación en el seno de cada asignatura está justificada. Por otro lado, se pone en evidencia la necesidad de concretar cómo se tratarán y evaluarán en cada asignatura, etapa y nivel educativo, proporcionando criterios claros sobre estos aspectos.

- La competencia es observable a través de sus componentes y, por tanto, los criterios (descriptores e indicadores) deben facilitar su observación.

- La competencia es perfectible, no se alcanza en un momento determinado, sino que se desarrolla de modo constante. Asimismo, las competencias clave sustentan futuras competencias más específicas en contextos de formación profesional y universitaria. La evaluación debe dar cuenta de este progreso, estructurando su adquisición en sucesivos niveles de logro por área, nivel educativo y etapa.

- La competencia obliga a adoptar un planteamiento educativo que complemente al tradicional enfoque centrado en el contenido y el docente. La pers- 
pectiva competencial aboga por un mayor protagonismo del alumno en el proceso de enseñanza, lo que conlleva impulsar la presencia del alumno en la evaluación a través de autoevaluaciones y coevaluaciones.

\section{EVALUAR POR COMPETENCIAS: CONCEPTO Y ESTADO DE LA CUESTIÓN}

Desde la aparición de las competencias en la Ley Orgánica 2/2006, de 3 de mayo, de Educación (LOE), todas las materias deben contribuir a este elemento curricular transversal. La actual modificación planteada por la Ley Orgánica 8/2013, de 9 de diciembre, para la mejora de la calidad educativa (LOMCE) ha refrendado el rol curricular de las competencias (Hortigüela, Abella y Pérez-Pueyo, 2015). Al respecto, para Rivas y Terroba (2010), la inclusión de las competencias en el currículo pretende: integrar los aprendizajes, favorecer que el alumnado asimile los contenidos, utilizándolos de manera efectiva cuando le resulten necesarios, y orientar la enseñanza al permitir identificar contenidos y criterios imprescindibles. En este sentido, la normativa presenta las competencias como los elementos que deben guiar la evaluación y promoción de los alumnos (artículos 21 y 28, LOE-LOMCE). No obstante, el hecho de adoptar una evaluación por competencias, no sólo se vincula con la evaluación del grado de adquisición de las mismas, sino, además, con el modo en que ésta se plantea.

En este línea, la complejidad y pluralidad conceptual en torno a la competencia también afecta al proceso evaluador (Zabala y Arnau, 2007). Por tanto, en primer lugar, parece de interés clarificar qué se entiende por evaluación por competencias. Siguiendo la línea de Pérez-Pueyo (2013), en esta propuesta se entiende la evaluación por competencias como aquella estrategia, Ilevada a cabo desde premisas formativas y auténticas, que valora el grado de adquisición de las competencias, siendo esta valoración el eje sobre el que tomar decisiones en el proceso de enseñanza (Bolívar, 2010; De Miguel, 2005; López-Pastor, 2009; Pepper, 2011; Yáñiz y Villardón, 2006). Para De Ketele y Gerard (2005), evaluar desde la perspectiva de las competencias supone proponer situaciones que necesitarán de una producción compleja del alumno para ser resuelta. Además, este enfoque de evaluación debe respetar una serie de postulados (Bolívar, 2010; De Miguel, 2005; López-Pastor, 2009; Pepper, 2011; Yáñiz y Villardón, 2006):

a) Evaluación auténtica. La evaluación plantea tareas que simulan problemas cercanos a la realidad del alumno, los cuales deben aplicar sus conocimientos para resolverlos. Situar la evaluación por competencias en el marco de la evaluación auténtica, supone enfatizar el valor del conocimiento contextualizado y en la acción.

b) Evaluación referida al criterio. La definición de un conglomerado de indicadores con sus respectivos niveles de logro se convierte en un paso previo e ineludible para determinar el grado de desarrollo de las competencias.

c) Los alumnos se "apoderan" de la evaluación. Se defiende el papel protagonista y activo del alumno en el proceso de enseñanza-aprendizaje y la evaluación compartida cobra mayor relevancia. La autoevaluación y la coevaluación ganan peso dentro del proceso.

d) Evaluación continua y formativa. Se debe informar de modo constante del grado de adquisición de las competencias, privilegiando el carácter procesual de 
la evaluación. Esta información debe servir para regular el proceso de enseñanza y aprendizaje. Bajo este planteamiento, se respeta el carácter evolutivo de las competencias, que no se aprenden, sino que se desarrollan con la experiencia.

Sin embargo, una década después de la aparición de este elemento curricular, se evidencia una reducida aplicación del modelo en las aulas (Monarca y Rappoport, 2013). En la Educación Física (EF) predomina el escepticismo, la desinformación y la falta de preparación para llevar a cabo este modelo (Barrachina y Blasco, 2012; Caballero, 2013; Zapatero-Ayuso, González-Rivera y Campos-Izquierdo, 2012). Si el desarrollo del modelo competencial constituye una preocupación relevante, la evaluación por competencias puede representar problema de mayor envergadura (Ramírez, 2016). A pesar de que los profesores de EF manifiestan una actitud tolerante y positiva hacia el modelo (Hortigüela et al., 2015), los estudios sugieren que: su evaluación no se desarrolla, no se establecen líneas de trabajo en los centros educativos para abordar el problema, no se proporcionan orientaciones y asesoramiento por parte de la administraciones y la normativa se percibe difusa y poco precisa (Barrachina y Blasco, 2012; Caballero, 2013; Hortigüela et al., 2015; Polo, 2010a).

En este contexto, este trabajo tiene como objetivo proporcionar una guía que sirva de referencia para abordar la problemática de la evaluación por competencias en EF. Para ello, se conceptualiza sobre el concepto competencia, sus implicaciones en la enseñanza y en una evaluación por competencias. Desde esta perspectiva, se presenta de forma general un modelo de evaluación en seis pasos (figura 1). Se ahondará

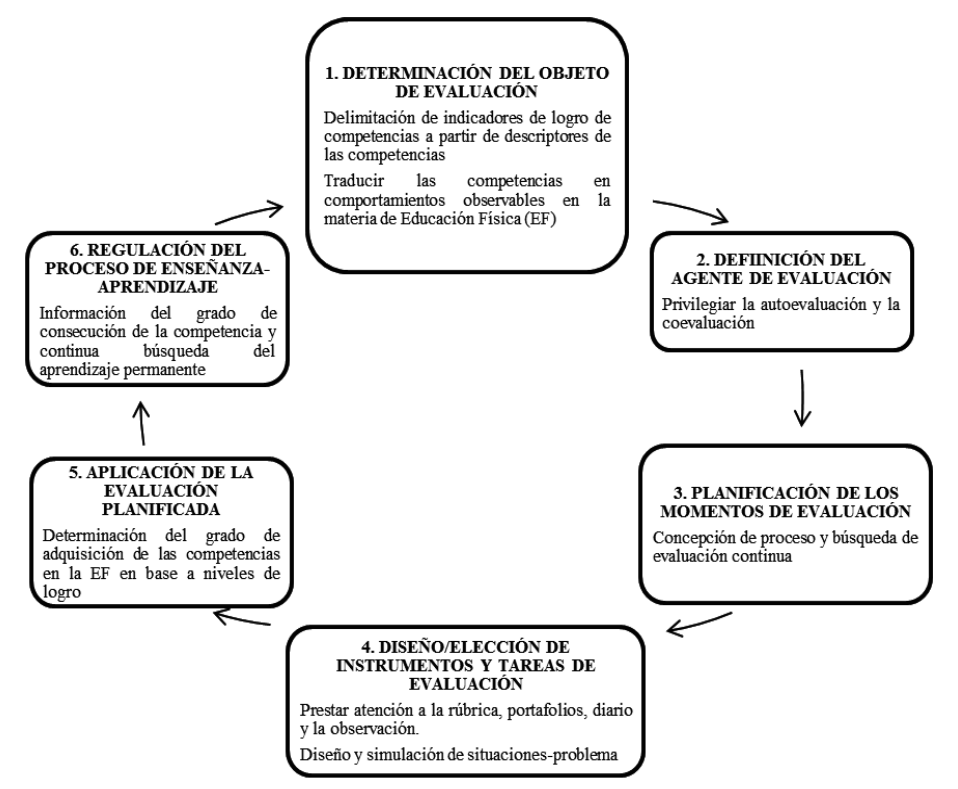

Figura 1. Un modelo para evaluar por competencias en Educación Física (Blázquez y Sebastiani, 2009; Bolívar, 2010; Escamilla, 2008; Escanero, Soria, Lafuente y

Ezquerra, 2007; López-Pastor, 1999, 2009; Tobón et al., 2006) 
en los cuatro primero pasos de este modelo. Así, se conceptualiza sobre el objeto de evaluación, el momento, el agente de evaluación y el diseño y/o elección de instrumentos. Basándose en el conocimiento y desarrollo de estos aspectos, se ofrece un procedimiento para diseñar un instrumento concreto (rúbrica), que permita evaluar por competencias en EF.

\section{El INDICADOR COMO REFERENTE PARA EVALUAR POR COMPETENCIAS}

Desde la perspectiva de la evaluación por competencias se exige una primera aproximación al conocimiento y comprensión de cada competencia en el marco de un centro educativo, un ciclo, un Departamento y/o un aula (Pérez-Pueyo, 2013). Para su comprensión se precisa atender a la normativa nacional (LOE-LOMCE y, concretamente, Orden ECD/65/2015) y supranacional (Consejo Europeo, 2006), al mismo tiempo que se pueden atender a las aportaciones sobre competencias en EF (por citar algunos, Blázquez y Sebastiani, 2009; Contreras y Cuevas, 2011; Ureña, 2010). Asimismo, no se debe obviar el conocimiento que se puede generar en un debate en el seno de un Departamento o Claustro para conocer y concretar las competencias (Pérez-Pueyo, 2013). Esta concreción curricular debe basarse en la definición de descriptores que darán lugar, a su vez, a la elaboración de sus correspondientes indicadores de logro.

Por un lado, se entiende por descriptor un enunciado en infinitivo que describe la competencia y la hace operativa para un centro, Departamento, nivel y/o grupo en una materia específica o en un centro educativo, de modo que permite vincular las competencias al resto de elementos curriculares, diseñar tareas para contribuir a su adquisición y definir indicadores para evaluarla (Blázquez y Sebastiani, 2009; Escamilla, 2008; Tobón et al., 2006).

Por otro lado, la terminología competencial ha dado lugar a una pluralidad terminológica para aludir al indicador de logro. En la literatura se pueden encontrar términos como marcadores de nivel, evidencias, competencias operativas o, más comúnmente, indicadores de desempeño, de logro o de dominio (Blázquez y Sebastiani, 2009; Moya y Luengo, 2011; Tobón et al., 2006; Ureña, 2010; Vázquez y Ortega, 2011). Concretamente, esta propuesta aboga por el término indicador de logro, pues pone de manifiesto el carácter evolutivo de la competencia, al mismo tiempo que revela el carácter continuo de su evaluación. En este sentido, se pueden definir diversos niveles de logro de cada indicador (tantos como un profesor o equipo docente considere), que informen del progreso del alumno sobre un descriptor, alejándose de posibles concepciones dicotómicas que informen únicamente sobre la consecución o no de la competencia.

Escamilla (2008) indica que los indicadores de desempeño son "enunciados que, respecto a una o varias competencias dadas, identifican un tipo de guía o patrón de conducta adecuado, eficaz, positivo (suponen siempre evolución y desarrollo). Proporcionan, al tiempo, una vía directa para determinar, de manera objetivable, el grado (cuantitativo y/o cualitativo) en que éstos se manifiestan." (p. 176). Específicamente en este trabajo, se concibe el indicador como un enunciado preciso que determina un comportamiento observable, a partir de un descriptor de competencia, para observar 


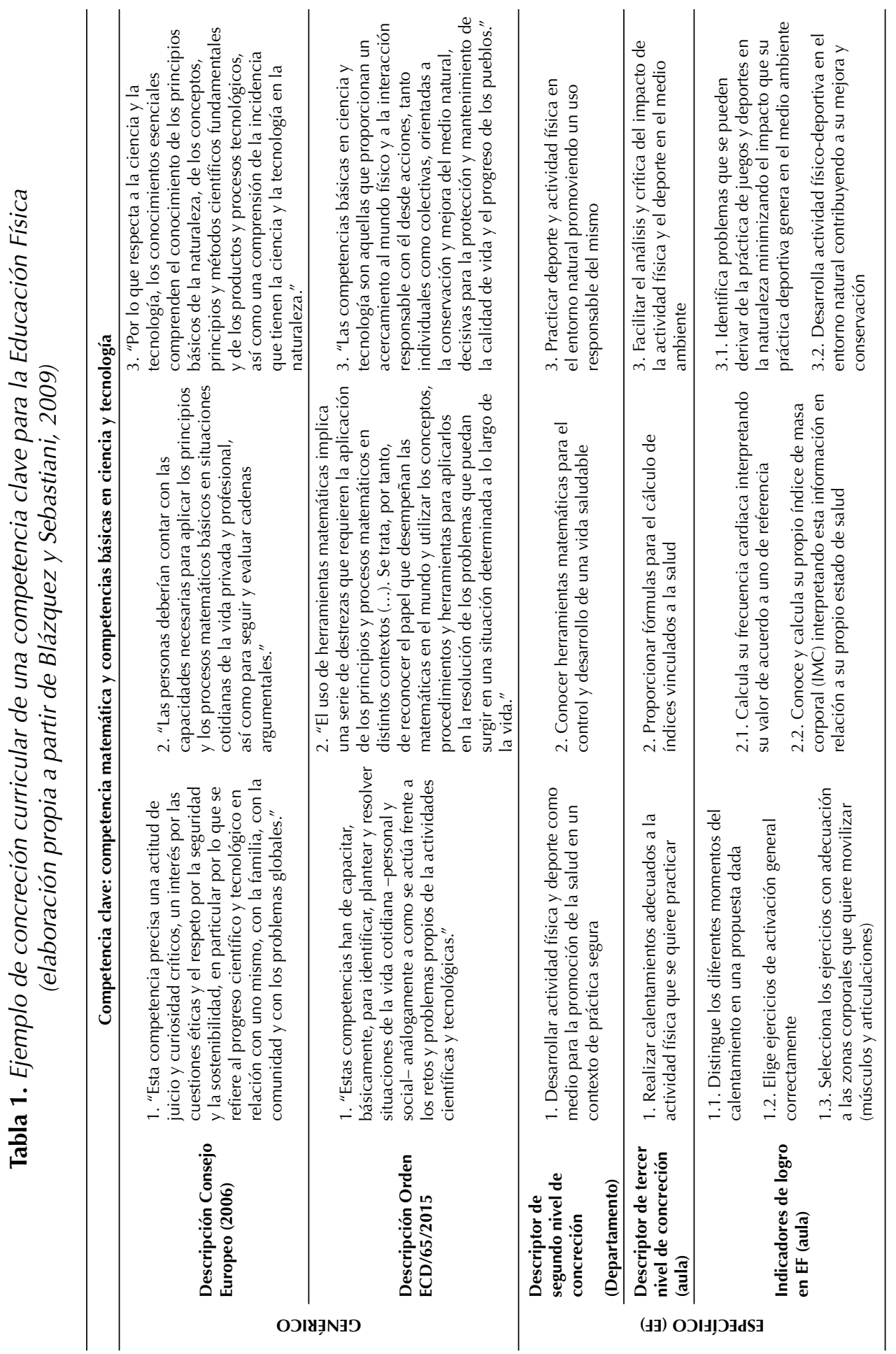


el desempeño del alumno en una situación problema y facilitar, por tanto, la evaluación del grado de adquisición (cuantitativo y/o cualitativo) de una determinada competencia (Blázquez y Sebastiani, 2009; Escamilla, 2008; Tobón et al., 2006). Para la formulación de indicadores de logro se recomienda que (Blázquez y Sebastiani, 2009): se expresen con verbos de acción en tercera persona del singular; se acompañen de los contenidos en los que se expresa el desarrollo de esa acción; y se refleje el contexto general en el que esperamos que el aprendizaje se aplique. Siguiendo a Escanero et al. (2007), se plantea una sencilla fórmula para redactar los indicadores de logro: verbo (conjugado) + objeto (contenidos) + condición (contexto e idoneidad).

De este modo, al finalizar este procedimiento se obtendrá un conglomerado de descriptores (que "describen" las competencias) y sus indicadores de logro correspondientes (que favorecerán la evaluación del grado de adquisición de la competencia). Al respecto, se da cuenta del carácter criterial de las competencias, que fue defendido en otras propuestas prácticas de enseñanza por competencias en EF (Lleixà, Torralba y Abrahão, 2010) y en otros sistemas educativos de reconocido prestigio sobre el modelo competencial (Ministère de l'Éducation, du Loisir et du Sport, 2009). Tanto los descriptores como los indicadores tomarán como referencia el nivel de concreción para el que se elaboran pudiendo presentarse para un centro educativo y común a todas las materias, para un Departamento o, concretamente, para un nivel o grupo en la EF. La tabla 1 recoge algunos ejemplos de concreción de la competencia matemática y competencias básicas en ciencia y tecnología para la EF jerárquicamente vinculados con la normativa europea y nacional. En ella se clarifica el modo propuesto para definir la competencia descrita genéricamente en el primer nivel de concreción curricular de forma operativa en el aula de EF (tercer nivel de concreción curricular).

\section{El momento y el agente de eVAlUaCión}

El planteamiento competencial nace como un modelo educativo que impulse "el aprendizaje permanente" (Consejo Europeo, 2006). Al respecto, entender el aprendizaje como un proceso "a lo largo de la vida" supone asumir el carácter continuo del mismo. De hecho, como se indicó en la conceptualización, la competencia se caracteriza por ser perfectible, desarrollándose de forma continua con la experiencia (Blázquez y Sebastiani, 2009; Zabala y Arnau, 2007). Asimismo, la aspiración educativa competencial es enseñar a que los alumnos sean más autónomos, para identificar aspectos que desarrollan bien y los que deben mejorar, así como aprender a gestionar su propia actividad física (Blázquez y Sebastiani, 2009). Por tanto, el fin del modelo competencial es emancipar al alumno para que sea capaz de actualizar, gestionar y aplicar el conocimiento de forma permanente en una sociedad globalizada y en constante cambio (Illeris, 2008). En este sentido, se pone de manifiesto la necesidad de plantear una evaluación por competencias continua e involucrar al alumno en su propia evaluación.

En cuanto al momento de la evaluación, además de las alusiones al carácter continuo y formativo de ésta (artículo 7 de la Orden ECD/65/2015), la literatura sobre el problema refrenda la necesidad de desarrollarla con una orientación procesual o con- 
tinua (López-Pastor, 2009). Siguiendo a este autor, la evaluación admite contemplar los tres momentos (inicial, continuo y final), siempre y cuando cada momento sirva a la comprensión, mejora y perfeccionamiento del proceso de enseñanza-aprendizaje y esté orientado al cambio. De este modo, la evaluación por competencias se convierte en un proceso constante de retroalimentación a los estudiantes sobre qué están mejorando en una determinada competencia y qué aspectos deben seguir mejorando a lo largo de un curso, ciclo o etapa educativa (Tobón et al., 2006).

En relación a la inclusión del alumno en la evaluación o evaluación compartida (López-Pastor, 1999), los estudiantes deben participar poco a poco en los procesos evaluativos a través de coevaluaciones y autoevaluaciones (Hortigüela, Abella y Pérez-Pueyo, 2014). De hecho, la capacidad de autoevaluación es imprescindible para alcanzar cierto nivel de independencia en la práctica de actividad física en el alumno, lo que podría dar lugar a un aprendizaje permanente en la EF (LópezPastor, 1999). Si bien la participación del alumno en la evaluación puede ser un asunto controvertido (Blázquez y Sebastiani, 2009), se han proporcionado algunas orientaciones para aplicarla (Tobón et al., 2006; Yáñiz y Villardón, 2006):

a) La autoevaluación también se aprende y los alumnos deben trabajarla.

b) Los docentes, como facilitadores, deben guiar a los estudiantes en su autoevaluación buscando que con ello tomen consciencia por sí mismo de sus logros, errores y puntos mejorables durante la adquisición de competencias.

c) Los estudiantes también pueden participar en la evaluación de las estrategias de evaluación (metaevaluación).

d) Se evalúa para mejorar y el profesorado debe resaltar su carácter formativo.

e) Se deben categorizar los aprendizajes. El alumno debe saber que no todos los aprendizajes tienen el mismo valor ni la misma incidencia en su proceso de adquisición de competencias.

f) No todo lo que está bien, está completo. El alumno debe saber lo que está bien, así como lo que le falta para llegar a los niveles de logro deseados.

No obstante, los agentes se diversifican en la evaluación por competencias. Por tanto, el profesor puede y debe participar de esta evaluación, complementando la visión del estudiante (Cano, 2008). Desde planteamientos formativos, la participación de diversos agentes favorece que la evaluación sea más objetiva y rigurosa (Díaz-Lucea, 2005; López-Pastor, 1999) En este sentido, el enfoque por competencias aboga por una evaluación que haga partícipe al alumno y se encuentre supervisada, intervenida y contrastada por el profesorado. De este modo, se responde a un replanteamiento de la relación profesor-alumno, convirtiendo al docente en un "facilitador de aprendizajes" (Gordon et al., 2009).

\section{LOS INSTRUMENTOS DE EVALUACIÓN Y EL PAPEL DE LA RÚBRICA EN LA EVALUACIÓN POR COMPETENCIAS}

En el marco de la evaluación formativa y por competencias, son muchos y variados los instrumentos a través de los cuales se pueden recoger evidencias que infor- 
men sobre el grado de consecución de una competencia. La didáctica de la EF ha contado con importantes referentes sobre las técnicas de evaluación en la materia (Blázquez, 1990; Díaz-Lucea, 2005; López-Pastor, 1999). Un instrumento de evaluación es el medio a través del cual se recaba y se registra información, evidencias, que van a favorecer la emisión de una valoración objetiva. Por tanto, las maneras de acceder a la información son amplias y escoger el instrumento adecuado en función de la evidencia que se quiera obtener resulta clave en el proceso de evaluación por competencias (López-Pastor, 2009). Algunos de los instrumentos más referenciados en el enfoque por competencias son los diarios de campo, la observación y las escalas de competencias o rúbricas (Blázquez y Sebastiani, 2009; Bolívar, 2010; Escamilla, 2008; Moya y Luengo, 2011).

En cuanto al uso del diario de campo o portafolios, no parece ser una iniciativa reciente, si bien parece haberse popularizado con la implantación del modelo por competencias. Para López-Pastor (2009) es una acumulación ordenada que contiene los registros o producciones de las actividades de aprendizaje realizadas por un alumno en un periodo de tiempo, que permite conocer el progreso del alumno respecto de aquellos aprendizajes. Según Blázquez y Sebastiani (2009) el uso del portafolios implica un procedimiento de recolección, selección, reflexión y proyección. Este proceso hace que sea especialmente útil para implicar al alumno en su aprendizaje, favorecer su participación, promover la reflexión y la autoevaluación y ofrecer información durante el propio proceso.

En lo que respecta a la observación, ésta conlleva centrar la atención en los implicados y su comportamiento en su relación con el entorno (Postic y De Ketele, 2000). No obstante, para observar es necesario: describir de forma precisa los comportamientos que se quieren observar, describir los indicadores y sus niveles de logro y propiciar las situaciones adecuadas (Blázquez y Sebastiani, 2009; Tobón et al., 2006). En consecuencia, este planteamiento exige centrar la atención en dos aspectos: la creación de las situaciones adecuadas y la elaboración de indicadores precisos y sus niveles de logro correspondientes.

En cuanto al diseño de tareas o situaciones, para determinar el grado en que un alumno ha desarrollado sus competencias se pueden diseñar tareas como la resolución de situaciones-problema, la realización de trabajos y actividades prácticas y/o las simulaciones (De Miguel, 2005; Instituto de Evaluación, 2009; Lleixà et al., 2010).

En lo que respecta a la definición de indicadores y niveles de logro, la rúbrica se presenta como un instrumento de notable importancia en la evaluación por competencias, recogiéndose como una prescripción normativa para evaluarlas (Orden ECD/65/2015). Este instrumento parece haberse erigido como un referente en la evaluación competencial siendo aplicado en diferentes contextos y niveles educativos (Blázquez y Sebastiani, 2009; Bolívar, 2010; Moya y Luengo, 2011; Tobón et al., 2006). Para Polo (2010b) la rúbrica "es una herramienta que se emplea para medir el nivel y la calidad de una tarea o una actividad" (p. 111). En este trabajo la rúbrica se entiende como una escala de clasificación descriptiva (Díaz-Lucea, 2005), en la que aparecen los indicadores que van a ser evaluados (primera columna izquierda) y sus respectivos niveles de logro definidos cualitativa o cuantitativamente (primera fila superior), que facilitarán la descripción de los 
indicadores en forma de comportamientos observables para determinar el grado de consecución con que sea manifestado el indicador (en las celdas inferiores). Como apunta Bolívar (2010), se trata de un instrumento pertinente en la evaluación por competencias, pues informa de la consecución o no de la competencia y el grado de desarrollo de la misma. Además, para Tobón et al. (2006) la rúbrica favorece la coevaluación y la autoevaluación dado que incluye los logros y los aspectos a mejorar y permite que el alumnado conozca de forma clara y precisa lo que se espera en su aprendizaje

\section{LA DETERMINACIÓN DE LOS NIVELES DE LOGRO Y EL PROCESO DE CONSTRUCCIÓN DE LA RÚBRICA}

La obtención de los indicadores, sobre la base de los descriptores, es el primer paso para la elaboración de los niveles de logro. El proceso de concreción de esos niveles en una escala cuantitativa o cualitativa dará lugar a la confección de una rúbrica. Asumiendo el carácter continuo, esta rúbrica debe plantearse con una concepción evolutiva que favorezca la concreción de niveles de logro cada vez más elevados en función del nivel educativo en el que se aplique. Si bien esta concreción debe seguir un proceso coherente y coordinado por todos los miembros de un centro educativo (Pérez-Pueyo, 2013), la elaboración de la misma puede llevarse a cabo de forma individual (por el profesor de EF en un nivel educativo), por un Departamento (mostrando el progreso competencial de los alumnos en EF a lo largo de una etapa educativa y sus niveles educativos) o a nivel de centro (Ilegando a acuerdos comunes a todas las asignaturas y niveles de logro para toda la etapa educativa). Algunos sistemas educativos como el de Quèbec utilizan procedimientos similares ofreciendo escalas que informan del grado de desarrollo de las competencias por cada ciclo educativo (Ministère de l'Éducation, du Loisir et du Sport, 2009). No obstante, la redacción de los indicadores y sus niveles de logro debe realizarse de acuerdo a decisiones sobre: el agente de evaluación (para el alumno o para el profesorado), el número de niveles de la escala (tres, cuatro, cinco, etc.) y su tipo, cualitativo o cuantitativo (figura 2).

Desde este planteamiento y tomando posición en el debate sobre la calificación y evaluación de las competencias, se aboga por la evaluación de las mismas, informando de su progreso y no requiriendo la obtención de una calificación (PérezPueyo, 2012). No obstante, si el docente, Departamento o Claustro, lo considerase necesario, se podría establecer un sistema de calificación de las mismas sobre la base de la rúbrica, indicando un porcentaje de calificación sobre cada indicador y nivel de logro, para obtener una calificación parcial, por indicador o competencia, o global, como calificación media de lo obtenido en las competencias evaluadas (Polo, 2010a).

A continuación, basados en la concreción de descriptores e indicadores de la competencia matemática y competencias básicas en ciencia y tecnología (tabla 1) y siguiendo el procedimiento de diseño de una rúbrica (figura 2), se expone un ejemplo concreto de ésta (tabla 2). Para su desarrollo, se ha tomado como referencia un descriptor concretado para el tercer nivel de concreción curricular de la citada 
competencia, cuyo enunciado es el siguiente: "1. Realizar calentamientos adecuados a la actividad física que se quiere practicar." Este descriptor fue concretado en tres indicadores de logro para su evaluación:

1.1. Distingue los diferentes momentos del calentamiento en una propuesta dada.

1.2. Elige ejercicios de activación general correctamente.

1.3. Selecciona los ejercicios con adecuación a las zonas corporales que quiere movilizar (músculos y articulaciones).

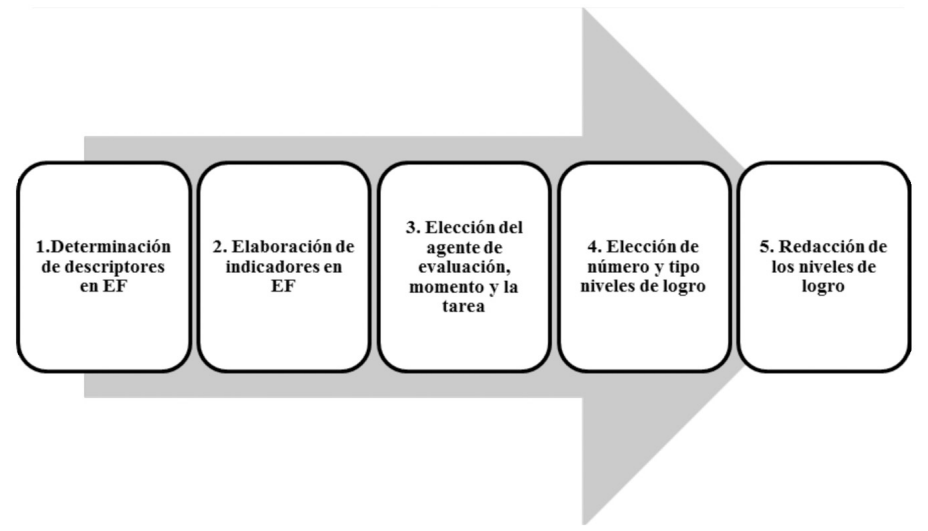

Figura 2. Pasos para la elaboración de una rúbrica en Educación Física.

Fuente: elaboración propia

Por tanto, la rúbrica propuesta se diseña a partir de estos indicadores. El agente de evaluación será el docente, que podrá observar el nivel de logro de los indicadores en una situación problema práctica, en la que un alumno debe guiar un calentamiento específico de forma autónoma. Estos indicadores han sido secuenciados en una escala de cinco niveles (desde nivel 1 o mínimo logro hasta nivel 5 o excelente). Estos niveles son redactados teniendo en cuenta que el agente de evaluación es el docente y, por tanto, incluyen vocabulario específico y técnico.

En el caso de optar por la aplicación de la rúbrica por parte de un alumno (autoevaluación o coevaluación), la redacción de indicadores y sus niveles de logro debería ser adecuada a las características de comprensión de los mismos. Además, se resalta que, en el ejemplo concreto de esta rúbrica, se han incluido tres indicadores y sus niveles de logro, pero podrían ser ampliados con más indicadores de este mismo descriptor o con indicadores de otras competencias que se quisieran evaluar. Asimismo, cabe resaltar que en esta propuesta no se especifica el nivel educativo en el que poner en práctica esta rúbrica, pues se pretende que sea un ejemplo para dar sentido al procedimiento para su diseño, poniendo el énfasis sobre el propio procedimiento y no en su producto. De este modo, se pretende dar respuesta al propósito del trabajo, proporcionando una referencia estructurada con la que abordar la problemática de la evaluación por competencias en el contexto particular de cada docente. 
Tabla 2. Ejemplo de rúbrica para la evaluación de un descriptor de competencia (adaptado de Blázquez y Sebastiani, 2009)

\begin{tabular}{|c|c|c|c|c|c|}
\hline \multicolumn{6}{|c|}{ Competencia clave: competencia matemática y competencias básicas en ciencia y tecnología } \\
\hline \multicolumn{6}{|c|}{ Dimensión de competencia: competencias básicas en ciencia y tecnología } \\
\hline \multicolumn{6}{|c|}{$\begin{array}{c}\text { Descriptor de competencia: } 1 . \text { Realizar calentamientos adecuados a la actividad física que se quiera } \\
\text { practicar }\end{array}$} \\
\hline & $\begin{array}{l}\text { Nivel } 1 \\
\text { (mínimo) }\end{array}$ & Nivel 2 (básico) & $\begin{array}{l}\text { Nivel } 3 \\
\text { (sofisticado) }\end{array}$ & $\begin{array}{l}\text { Nivel } 4 \\
\text { (avanzado) }\end{array}$ & $\begin{array}{l}\text { Nivel } 5 \\
\text { (Excelente) }\end{array}$ \\
\hline $\begin{array}{l}\text { Indicador de } \\
\text { logro: } 1.1 . \\
\text { Distingue los } \\
\text { diferentes } \\
\text { momentos del } \\
\text { calentamiento } \\
\text { en una } \\
\text { propuesta dada }\end{array}$ & $\begin{array}{l}\text { Sólo distingue } \\
\text { momentos del } \\
\text { calentamiento } \\
\text { ocasionales }\end{array}$ & $\begin{array}{l}\text { Distingue con } \\
\text { dificultad los } \\
\text { diferentes } \\
\text { momentos en el } \\
\text { calentamiento }\end{array}$ & $\begin{array}{l}\text { Distingue cada } \\
\text { momento del } \\
\text { calentamiento }\end{array}$ & $\begin{array}{l}\text { Distingue } \\
\text { bien los } \\
\text { momentos del } \\
\text { calentamiento } \\
\text { y los tipos de } \\
\text { ejercicios que se } \\
\text { relacionan con } \\
\text { cada momento }\end{array}$ & $\begin{array}{l}\text { Distingue } \\
\text { perfectamente } \\
\text { los momentos } \\
\text { del } \\
\text { calentamiento } \\
\text { y sus ejercicios } \\
\text { y justifica la } \\
\text { estructura } \\
\text { de un } \\
\text { calentamiento }\end{array}$ \\
\hline $\begin{array}{l}\text { Indicador de } \\
\text { logro: } 1.2 \text {. } \\
\text { Elige ejercicios } \\
\text { de activación } \\
\text { general } \\
\text { correctamente }\end{array}$ & $\begin{array}{l}\text { No conoce } \\
\text { ejercicios de } \\
\text { activación } \\
\text { general de } \\
\text { calentamiento }\end{array}$ & $\begin{array}{l}\text { Conoce pocos } \\
\text { ejercicios de } \\
\text { activación } \\
\text { general, pero } \\
\text { no los aplica } \\
\text { adecuadamente } \\
\text { en una } \\
\text { secuencia de } \\
\text { calentamiento }\end{array}$ & $\begin{array}{l}\text { Conoce } \\
\text { variados } \\
\text { ejercicios de } \\
\text { activación } \\
\text { general, pero } \\
\text { no los aplica } \\
\text { coherentemente } \\
\text { en un } \\
\text { calentamiento }\end{array}$ & $\begin{array}{l}\text { Conoce una } \\
\text { amplia gama } \\
\text { de ejercicios } \\
\text { de activación } \\
\text { general, pero } \\
\text { los desarrolla } \\
\text { alternativamente } \\
\text { con ejercicios } \\
\text { específicos sin } \\
\text { estructurarlos } \\
\text { en un } \\
\text { calentamiento }\end{array}$ & $\begin{array}{l}\text { Conoce una } \\
\text { amplia gama } \\
\text { de ejercicios } \\
\text { de activación } \\
\text { y los distingue } \\
\text { de ejercicios } \\
\text { específicos, } \\
\text { así como los } \\
\text { estructura } \\
\text { correctamente } \\
\text { en un } \\
\text { calentamiento }\end{array}$ \\
\hline $\begin{array}{l}\text { Indicador de } \\
\text { logro: } 1.3 \text {. } \\
\text { Selecciona los } \\
\text { ejercicios con } \\
\text { adecuación } \\
\text { a las zonas } \\
\text { corporales } \\
\text { que quiere } \\
\text { movilizar } \\
\text { (músculos y } \\
\text { articulaciones) }\end{array}$ & $\begin{array}{l}\text { No conoce } \\
\text { las partes } \\
\text { corporales } \\
\text { que quiere } \\
\text { calentar ni } \\
\text { ejercicios } \\
\text { para su } \\
\text { calentamiento }\end{array}$ & $\begin{array}{l}\text { Conoce } \\
\text { ejercicios de } \\
\text { calentamiento, } \\
\text { pero no } \\
\text { identifica } \\
\text { las partes } \\
\text { corporales que } \\
\text { intervienen en } \\
\text { los ejercicios } \\
\text { del mismo }\end{array}$ & $\begin{array}{l}\text { Conoce y } \\
\text { desarrolla } \\
\text { ejercicios } \\
\text { para movilizar } \\
\text { amplias partes } \\
\text { corporales } \\
\text { (extremidades } \\
\text { y tronco), sin } \\
\text { identificar } \\
\text { músculos o } \\
\text { articulaciones }\end{array}$ & $\begin{array}{l}\text { Conoce y aplica } \\
\text { ejercicios para } \\
\text { movilizar y } \\
\text { estirar algunos } \\
\text { músculos y } \\
\text { articulaciones } \\
\text { concretos de las } \\
\text { extremidades y } \\
\text { el tronco }\end{array}$ & $\begin{array}{l}\text { Conoce } \\
\text { y nombra } \\
\text { músculos y } \\
\text { articulaciones } \\
\text { de tronco y } \\
\text { extremidades } \\
\text { y desarrolla } \\
\text { ejercicios para } \\
\text { movilizar y } \\
\text { estirar con } \\
\text { precisión estos } \\
\text { músculos y } \\
\text { articulaciones }\end{array}$ \\
\hline
\end{tabular}

\section{Conclusiones}

El hecho de incluir el concepto de competencia en los desarrollos curriculares y planteamientos educativo conlleva asumir algunas de las características de este término. Las competencias se manifiestan en la movilización y aplicación de saberes (conceptuales, procedimentales y actitudinales) en situaciones concretas. Además, las competencias son elementos curriculares que se perfeccionan y desarrollan constantemente en las sucesivas etapas educativas. Por tanto, para evaluar competencias se hace preciso 
responder a dos aspectos: su carácter observable y su desarrollo continuo. Este hecho obliga a plantear estrategias evaluativas formativas, con carácter continuo y que involucren al alumno, y auténticas, basadas en criterios observables en situaciones-problema.

Esta propuesta ha puesto de manifiesto la necesidad de conocer las competencias (a través de los currículos y/o fuentes bibliográficas), reflexionar sobre cómo se va a contribuir en un área particular y, en consecuencia, evaluar su adquisición. Esta reflexión y acción crítica garantiza la obtención de unos criterios claros, precisos y observables (descriptores e indicadores) que orienten el trabajo competencial en el aula y faciliten su evaluación.

Este esfuerzo de concreción puede responder al carácter criterial y funcional de la competencia, al mismo tiempo que parece convertir este elemento curricular transversal en un elemento operativo en una materia concreta. Ahora bien, dado su carácter perfectible, se debe conocer en qué nivel se adquiere una determinada competencia. Esta propuesta aboga por la rúbrica como un instrumento que permite evidenciar el grado de adquisición de los criterios (indicadores), estableciendo y secuenciando con precisión el logro de los mismos. Por tanto, la rúbrica parece responder al carácter observable, criterial y perfectible de la competencia.

No obstante, la normativa no ofrece referencias claras sobre cómo incluir las competencias y la evaluación de las mismas en la EF u otras materias, por lo que se pone énfasis en la necesidad de que el profesorado se implique, reflexione y conceptualice sobre las mismas y la evaluación para conseguir implantar procesos de evaluación por competencias.

\section{REFERENCIAS BIBLIOGRÁFICAS}

Baartman, L. y Bruijn, E. (2011). Integrating knowledge, skills and attitudes: Conceptualizing learning processes towards vocational competence. Educational Research Review, 6, 125-134.

Barrachina, J. y Blasco, J. E. (2012). Análisis del desarrollo de las competencias básicas en el currículum de la Educación Física en la ESO en la Marina Baixa. Un estudio de caso. Apunts: Educación física y deportes, 110, 36-44. http://doi. org/10.5672/apunts.2014-0983.es.(2012/4).110.04.

Blázquez, D. (1990). Evaluar en Educación Física. Barcelona: INDE.

Blázquez, D. y Sebastiani, E. M. (2009). Enseñar por competencias en Educación Física. Barcelona: INDE.

Bolívar, A. (2010). Competencias básicas y Currículum. Síntesis: Madrid.

Caballero, J. A. (2013). La contribución del área de la Educación Física a las competencias básicas: opinión de los docentes. Emásf, Revista Digital de Educación Física, 4(21), 1-18.

Cano, M. E. (2008). La evaluación por competencias en la educación superior. Profesorado. Revista de currículum y formación del profesorado, 12(3). Recuperado de http://www.ugr.es/local/recfpro/rev123COL1.pdf.

Consejo Europeo (2006). Recomendación del parlamento europeo y del consejo de 18 de diciembre de 2006 sobre las competencias clave para el aprendizaje permanente. Diario Oficial de Europa, L394/10, de 30/12/2006, 10-18. 
Contreras, O. y Cuevas, R. (2011). Las competencias básicas desde la Educación Física. Barcelona: INDE.

De Ketele, J. M., y Gerard, F. M. (2005). La validation des épreuves d'évaluation selon l'approche par les compétences. Mesure et évaluation en éducation, 28(3), $1-26$.

De Miguel, M. (2005). Modalidades de enseñanza centradas en el desarrollo de competencias. Orientaciones para promover el cambio metodológico en el Espacio Europeo de Educación Europeo. Oviedo: Universidad de Oviedo.

Díaz-Lucea, J. (2005). La evaluación formativa como instrumento de aprendizaje en educación física. Barcelona: INDE.

Ellström, P. E. y Kock, H. (2008). Competence Development in the Workplace: Concepts, Strategies and Effects. Asia Pacific Education Review, 9(1), 5-20.

Escamilla, A. (2008) Las competencias básicas. Claves y propuestas para su desarroIlo en los centros. Barcelona: GRAO.

Escanero, J. F., Soria, M., Lafuente, J. V. y Ezquerra, L. (2007). Formular y evaluar competencias. Zaragoza: Prensas Universitarias de Zaragoza.

Gordon, J., Halász, G., Krawczyk, M., Leney, T., Michel, A., Pepper, D., ... Wisniewski, J. (2009). Key Competences in Europe: Opening Doors for Lifelong Learns across the School Curriculum and Teacher Education. Warsaw: CASE (Center for Social and Economic Research).

Hortigüela, D., Abella, V. y Pérez-Pueyo, A. (2014). ¿Trabajamos para evaluar las Competencias Básicas? Estudio de la percepción del profesorado sobre la implantación en los centros educativos. Revista de evaluación educativa, 3(1). Recuperado de http://revalue.mx/revista/index.php/revalue/article/view/101.

Hortigüela, D., Abella, V. y Pérez-Pueyo, A. (2015). ¿Se han implantado las competencias básicas en los centros educativos? Un estudio mixto sobre su programación como herramienta de aprendizaje. Revista Iberoamericana de Evaluación Educativa, 8(1), 177-192.

Illeris, K. (2008). Competence Development -the key to modern education, or just another buzzword? Asia Pacific Education Review, 9(1), 1-4.

Instituto de Evaluación (2009). Evaluación general de diagnóstico 2009. Marco de evaluación. Madrid: Ministerio de Educación.

Ley Orgánica 2/2006, de 3 de mayo, de Educación. Boletín Oficial del Estado de 4 de Mayo de 2006.

Ley Orgánica 8/2013, de 9 de diciembre, para la mejora de la calidad educativa. Boletín Oficial del Estado de 10 de Diciembre de 2013.

Lleixà, T., Torralba, M. A. y Abrahão, S. R. (2010). Evaluación de competencias en Educación Física: Investigación-acción para el diseño de procedimientos de evaluación en la Etapa Primaria. Movimento, 16(4), 33-51.

López-Pastor, V. (1999). Prácticas de evaluación en Educación Física: Estudio de casos en Primaria, Secundaria y Formación del Profesorado. Valladolid: Universidad de Valladolid.

López-Pastor, V. (2009). Evaluación formativa y compartida en Educación Superior. Propuestas, técnicas, instrumentos y experiencias. Madrid: Narcea. 
Ministère de l'Éducation, du Loisir et du Sport (2009). Scales of competency levels secondary school education cycle two. Physical Education and Health (Third edition, Secondary III, IV and V). Quèbec: Gouvernement du Quèbec. Recuperado de http://collections.banq.qc.ca/ark:/52327/bs1958973.

Monarca, H. y Rappoport, S. (2013). Investigación sobre los procesos de cambio educativo: El caso de las competencias básicas en España. Revista de Educación, Extraordinario, 54-78. http://doi.org/10.4438/1988-592X-RE-2013-EXT-256.

Moya, J. y Luengo, F. (2011). Teoría y práctica de las competencias básicas. Barcelona: GRAO.

Orden ECD/65/2015, de 21 de enero, por la que se describen las relaciones entre las competencias, los contenidos y los criterios de evaluación de la educación primaria, la educación secundaria obligatoria y el bachillerato. Boletín Oficial del Estado de 29 de enero de 2015.

Pérez-Pueyo, A. (2012). Las competencias básicas en EF. ¿Evaluación o calificación? Revista Española de Educación Física y Deportes, 397, 37-49.

Pérez-Pueyo, A. (2013). Programar y evaluar competencias básicas en 15 pasos. Barcelona: GRAO.

Perrenoud, P. (2004) Diez nuevas competencias para enseñar. Barcelona: GRAO.

Polo, I. (2010a). La evaluación de las competencias básicas. Avances en Supervisión Educativa. Revista de la Asociación de Inspectores de Educación de España, 12. Recuperado de http://www.adide.org/revista/index.php/ase/article/view/440.

Polo, I. (2010b). Las rúbricas como instrumentos de apoyo. La evaluación de las competencias básicas en Educación Física. Tándem. Didáctica de la Educación Física, 32, 106-119.

Postic, M. y De Ketele, J. M. (2000). Observar las situaciones educativas. Madrid: Narcea.

Ramírez, A. (2016). Evaluación de las competencias básicas en educación primaria: una mirada desde la óptica docente. Profesorado. Revista de currículum y formación del profesorado, 20(1). Recuperado de http://www.ugr.es/local/recfpro/ rev201COL1.pdf.

Rieckmann, M. (2012). Future-oriented higher education: Which key competencies should be fostered through university teaching and learning? Futures, 44, 127-135.

Rivas, E. y Terroba, J. (2010). Desarrollo de la competencia básica "Comunicación lingüística" a través del cuento motor y el cuaderno del alumno en la asignatura de Educación Física. Contextos educativos. Revista de Educación, 13, 155-174. http://doi.org/10.18172/con.632.

Rychen, D. y Salganik, L. (2004). Definir y seleccionar las competencias fundamentales para la vida. México D.F.: Fondo de Cultura Económica.

Tejada, J. (2012). La alternancia de contextos para la adquisición de competencias profesionales en escenarios complementarios de educación superior: marco y estrategia. Educación XXI, 15(2), 19-40.

Tiana, A., Moya, J. y Luengo, F. (2011). Implementing Key Competences in Basic Education: reflections on curriculum design and development in Spain. European Journal of Education, 46(3), 307-322. 
Tobón, S., Rial, A., Carretero, M. y García, J. (2006). Competencias, calidad y educación superior. Bogotá: Alma Mater Magisterio.

Ureña, F. (2010). La Educación Física en Secundaria basada en competencias. Proyecto curricular y programación. Barcelona: INDE.

Vázquez, P. y Ortega, J. L. (2011). Competencias básicas. Desarrollo y evaluación en Educación Secundaria. Madrid: Wolters Kluwer.

Yáñiz, C. y Villardón, L. (2006). Planificar desde competencias para promover el aprendizaje. El reto de la sociedad del conocimiento para el profesorado universitario. Cuadernos monográficos del ICE, 12. Bilbao: Deusto Digital.

Zabala, A. y Arnau, L. (2007). 11 ideas clave: cómo aprender y enseñar competencias. Barcelona: GRAO.

Zapatero-Ayuso, J. A., González-Rivera, M. D. y Campos-Izquierdo, A. (2012). La formación de los docentes de Educación Física en torno a la enseñanza por competencias a través de un grupo de discusión. Emásf, Revista Digital de Educación Física, 3(17), 1-15. 